\title{
Preliminary research on abnormal brain detection by wavelet-energy and quantum- behaved PSO
}

\author{
Yudong Zhang ${ }^{\mathrm{a}, \mathrm{b}, *}$, Genlin Ji ${ }^{\mathrm{a}, \mathrm{b}}$, Jiquan Yang ${ }^{\mathrm{b}}$, Shuihua Wang ${ }^{\mathrm{a}, \mathrm{b}, *}$, Zhengchao Dong ${ }^{\mathrm{c}}$, \\ Preetha Phillips ${ }^{\mathrm{d}}$ and Ping Sun ${ }^{\mathrm{e}}$ \\ ${ }^{a}$ School of Computer Science and Technology, Nanjing Normal University, Nanjing, Jiangsu, China \\ ${ }^{\mathrm{b}}$ Jiangsu Key Laboratory of 3D Printing Equipment and Manufacturing, Nanjing, Jiangsu, China \\ ${ }^{\mathrm{c}}$ Translational Imaging Division and MRI Unit, Columbia University and New York State Psychiatric \\ Institute, New York, NY, USA \\ ${ }^{\mathrm{d}}$ School of Natural Sciences and Mathematics, Shepherd University, Shepherdstown, WV, USA \\ ${ }^{\mathrm{e}}$ Department of Electrical Engineering, The City College of New York, CUNY, New York, NY, USA
}

\begin{abstract}
It is important to detect abnormal brains accurately and early. The wavelet-energy (WE) was a successful feature descriptor that achieved excellent performance in various applications; hence, we proposed a WE based new approach for automated abnormal detection, and reported its preliminary results in this study. The kernel support vector machine (KSVM) was used as the classifier, and quantum-behaved particle swarm optimization (QPSO) was introduced to optimize the weights of the SVM. The results based on a $5 \times 5$-fold cross validation showed the performance of the proposed WE + QPSO-KSVM was superior to "DWT + PCA + BP-NN", "DWT + PCA + RBF-NN", "DWT + PCA + PSO-KSVM", "WE + BPNN", "WE + KSVM", and "DWT + PCA + GA-KSVM" w.r.t. sensitivity, specificity, and accuracy. The work provides a novel means to detect abnormal brains with excellent performance.
\end{abstract}

Keywords: Magnetic resonance imaging, particle swarm optimization, quantum-behaved PSO, wavelet energy

\section{Introduction}

It remains a hot topic to develop novel systems for automatic pre-clinical diagnosis for magnetic resonance (MR) images [1-3]. Traditional manual methods are costly, tedious, time-consuming, and irreproducible, due to the tremendous and massive amount of data. This makes it necessary to develop automatic computer-aided diagnosis (CAD) tools [4].

The most discriminant features of a normal brain is its symmetry in either the axial or the coronal direction. Usually the asymmetry along an axial or coronal MR brain suggests a pathological brain. The characteristic of the symmetry-asymmetry was modelled by a mass of image preprocessing and proprocessing techniques, and was commonly used to detect pathological brains [5].

\footnotetext{
${ }^{*}$ Corresponding author: Yudong Zhang, Shuihua Wang, School of Computer Science and Technology, Nanjing Normal University, Nanjing, Jiangsu 210023, China. E-mail: zhangyudong@njnu.edu.cn; wangshuihua@njnu.edu.cn.
}

0928-7329/16/\$35.00 @ 2016 - IOS Press and the authors. All rights reserved This article is published online with Open Access and distributed under the terms of the Creative Commons Attribution NonCommercial License. 
In the last decade, various CAD systems were proposed for brain MR image classification. ElDahshan, Hosny and Salem [6] extracted the approximation and detail coefficients of 3-level discrete wavelet transform (DWT), reduced them by the classical feature-reduction method of principal component analysis (PCA), and then used feed-forward back-propagation artificial neural network (FP-ANN) and $\mathrm{K}$-nearest neighbor (KNN) as the classifiers. Zhang et al. [7] followed the feature extraction and reduction mechanism, but used a feed-forward neural network (FNN) with scaled chaotic artificial bee colony (SCABC) as the classifier. Subsequently, Zhang et al. [8] suggested to replace SCABC with scaled conjugate gradient (SCG) method, and they found the results of SCG were better than existing methods. Ramasamy and Anandhakumar [9] used a totally different method, i.e., they employed a fast-Fourier-transform based expectation maximization Gaussian mixture model for brain tissue classification. Zhang and $\mathrm{Wu}$ [10] proposed to use kernel support vector machine (KSVM), and they suggested three new kernels. Saritha et al. [11] was the first to use a novel feature of wavelet-entropy (WE), and employed spider-web plots (SWP) to further reduce features. Afterwards, they used the probabilistic neural network (PNN) to detect pathological brains. Zhang et al. [12] suggested that the classification performance did not change even if removing the procedure of SWP. Das et al. [13] proposed to use Ripplet transform (RT) + PCA + least square SVM (LS-SVM), and the $5 \times 5$ CV showed high classification accuracies. Kalbkhani et al. [14] modelled the detail coefficients of 2-level DWT by generalized autoregressive conditional heteroscedasticity $(\mathrm{GARCH})$ statistical model, and the parameters of GARCH model are considered as the primary feature vector. Their classifier was chosen as KNN and support vector machine (SVM) models. Zhang et al. [15] suggested to use particle swarm optimization (PSO) to train the KSVM, and their result on a 90 image database achieved $97.11 \%$ accuracy. Padma and Sukanesh [16] used combined wavelet statistical texture features, to segment and classify AD benign and malignant tumor slices. El-Dahshan et al. [17] used the feedback pulse-coupled neural network for image segmentation, the DWT for features extraction, the PCA for reducing the dimensionality of the wavelet coefficients, and the FBPNN to classify inputs into normal or abnormal. Zhou et al. [18] used wavelet-entropy as the feature space, then they employed a Naive Bayes classifier (NBC) classification method. Their results over 64 images showed that the sensitivity of the classifier is $94.50 \%$, the specificity $91.70 \%$, the overall accuracy $92.60 \%$. Zhang et al. [19] used discrete wavelet packet transform (DWPT), and harnessed Tsallis entropy to obtain features from DWPT coefficients. Then, they used a generalized eigenvalue proximal SVM (GEPSVM) with radial basis function (RBF) kernel. Wang et al. [20] proposed a combination of GA and KSVM to solve this problem. Yang et al. [21] selected wavelet-energy as the features, and introduced biogeography-based optimization (BBO) to train the SVM. Wang et al. [22] suggested to use stationary wavelet transform (SWT) to replace DWT, and then they proposed a hybridization of PSO and ABC (HPA) algorithm to train the classifier. Zhang et al. [23] suggested to use a 3D eigenbrain method to detect subjects and brain regions related to AD. The accuracy achieved $92.36 \pm 0.94$.

All above-mentioned methods achieved promising results; however, there are two problems. (1) Most of them used DWT, whose coefficients require a large storage memory and (2) Most of them used ANN or SVM. Current training algorithm cannot guarantee that the solutions are optimal.

To address these two problems, we gave two potential contributions in this study. First, we introduced the wavelet energy (WE), which is a rather novel feature that calculates the energy from the waveletsubband coefficients. It has already been applied to many academic and industrial fields. Next, we proposed to use the quantum-behaved particle swarm optimization (QPSO) method to train the kernel SVM (KSVM), with the aim of obtaining the global optimal weights of the classifier. 


\section{Materials}

The datasets brain consists of ninety MR brain images (T2-weighted), scanned in axial plane and $256 \times 256$ resolution, which can be downloaded from the homepage of Harvard Medical School (URL: http://www.med.harvard.edu/aanlib/home.html). The dataset consist of normal brains and 17 diseases including Glioma, Metastatic adenocarcinoma, Metastatic bronchogenic carcinoma, Meningioma, Sarcoma, Alzheimer, Huntington, Motor Neuron disease, Cerebral Calcinosis, Pick's disease, Alzheimer plus visual agnosia, Multiple sclerosis, AIDS dementia, Lyme encephalopathy, Herpes encephalitis, Creutzfeld-Jakob disease, and Cerebral Toxoplasmosis. Note that all diseases are treated as abnormal brains, and our task is a binary classification problem, i.e., to detect abnormal brains from normal brains.

We randomly selected 5 images for each type of brain. Since in the dataset there are normal brains and 17 diseases of abnormal brains, $5 *(1+17)=90$ images was selected to construct the brain dataset, consisting of 5 normal and 85 abnormal brain images in total.

In this study, a $5 \times 5$-fold $\mathrm{CV}$ was implemented, with the aim of reducing randomness. The confusion matrices of 5 runs were combined to form the final confusion matrix, with the ideal results of 425 abnormal instances and 25 normal instances perfectly recognized.

\section{Methodology}

\subsection{Wavelet-energy}

Wavelet coefficients from the four subbands (LL, HL, LH, and HH) of all decomposition levels [24] were used to formulate the features used in this study. They are defined as:

$$
E(S)=\sum_{x} \sum_{y} S(x, y)^{2}, S \in[\mathrm{LL}, \mathrm{HL}, \mathrm{LH}, \text { and } \mathrm{HH}]
$$

where $S$ represents a specific subband and $E$ the wavelet-energy. These energies reflect the strength of the images' details in different subbands. Suppose a 3-level decomposition, the final extracted features are the energies from the 10 subbands including (LL3, LH3, HL3, HH3, LH2, HL2, HH2, LH1, HL1, HH1). Haar wavelet was employed as it was widely used and had successful results [25].

\section{2. $S V M$}

Suppose the sample is a $p$-dimensional data vector, and our task is to create a $(p-1)$-dimensional hyperplane. We choose the hyperplane to maximize the distance from it to the nearest data point on each class [26].

Given a $p$-dimensional $N$-size training dataset of the form

$$
\left\{\left(x_{n}, y_{n}\right) \mid x_{n} \in R^{p}, y_{n} \in\{-1,+1\}\right\}, n=1, \ldots, N
$$

where $x_{n}$ is a $p$-dimensional vector, and $y_{n}$ is either -1 or +1 corresponds to the class 1 or 2 . Considering that any hyperplane can be written in the form of $\mathbf{w} \mathbf{x}-b=0$, where $\mathbf{w}$ and $b$ represents the weights and biases of the classifier. Hence, we define the two parallel hyperplanes by the equations as $\mathbf{w x}-b= \pm$ 1. Afterwards, the problem is finalized as

$$
\min _{\mathbf{w}, b} \frac{1}{2}\|\mathbf{w}\|^{2} \text { s.t. } y_{n}\left(\mathbf{w} x_{n}-b\right) \geqslant 1, n=1, \ldots, N
$$


In practical applications, there may is no hyperplane that splits the samples perfectly. In such case, the "soft margin" method was introduced since it chooses a hyperplane that splits the given samples in a "soft" way other than in a "hard" way.

Positive slack vector $\boldsymbol{\xi}=\left(\xi_{1}, \ldots, \xi_{n}, \ldots, \xi_{N}\right)$ are addeded to measure the misclassification degree of sample $x_{n}$. Then, the optimal hyperplane can be obtained by solving:

$$
\min _{\mathbf{w}, \xi, \mathbf{b}} \frac{1}{2}\|\mathbf{w}\|^{2}+c e^{T} \boldsymbol{\xi} \text { s.t. }\left\{\begin{array}{l}
y_{n}\left(\mathbf{w}^{T} x_{n}-\mathbf{b}\right) \geqslant 1-\xi_{n} \\
\xi_{n} \geqslant 0
\end{array}, n=1, \ldots, N\right.
$$

where $C$ is the error penalty (i.e., box constraint in some other literatures) and $e$ is a vector of ones of $N$-dimension. Therefore, the problem can be solved using "Lagrange multiplier" as

$$
\min _{\mathbf{w}, \xi, \mathbf{b}} \max _{\alpha, \beta}\left\{\frac{1}{2}\|\mathbf{w}\|^{2}+c e^{T} \boldsymbol{\xi}-\sum_{n=1}^{N} \beta_{n} \xi_{n}-\sum_{n=1}^{N} \alpha_{n}\left[y_{n}\left(\mathbf{w}^{T} x_{n}-\mathbf{b}\right)-1+\xi_{n}\right]\right\}
$$

The min-max problem is not easy to solve, so dual form technique is commonly proposed to solve it as

$$
\min _{\alpha} \sum_{n=1}^{N} \alpha_{n}-\frac{1}{2} \sum_{n=1}^{N} \sum_{m=1}^{N} \alpha_{m} \alpha_{n} y_{m} y_{n} x_{m}^{T} x_{n} \quad \text { s.t. }\left\{\begin{array}{l}
0 \leqslant \alpha_{n} \leqslant C \\
\sum_{n=1}^{N} \alpha_{n} y_{n}=0 \quad, n=1, \ldots, N
\end{array}\right.
$$

The key advantage is that the slack variables $\xi_{n}$ vanish from the dual problem, with the constant $C$ appearing only as an additional constraint on the Lagrange multipliers.

\subsection{Kernel $S V M$}

Linear SVMs have the downside to linear hyperplane, which cannot classify complicated distributed data. The kernel SVM algorithm is a variant of SVM, except the dot product in SVM is replaced with a nonlinear kernel function.

The procedures of KSVMs are basically to fit the maximum-margin hyperplane in a transformed higher dimensional feature space. For each kernel, there usually is one or more parameters so as to make the kernel flexible and tailor itself to practical data. RBF kernel was chosen due to its superior performances to other kernels reported in a mass of open literatures [27]. RBF is defined with the form of

$$
k\left(x_{m}, x_{n}\right)=\exp \left(-\frac{\left\|x_{m}-x_{n}\right\|}{2 \sigma^{2}}\right)
$$

where $\sigma$ represents the scaling factor, and $k$ the kernel function. Put Eq. (7) into Eq. (6), and we got the final SVM training function as

$$
\begin{aligned}
& \min _{\alpha} \sum_{n=1}^{N} \alpha_{n}-\frac{1}{2} \sum_{n=1}^{N} \sum_{m=1}^{N} \alpha_{m} \alpha_{n} y_{m} y_{n} \exp \left(-\frac{\left\|x_{m}-x_{n}\right\|}{2 \sigma^{2}}\right) \\
& \text { s.t. }\left\{\begin{array}{l}
0 \leqslant \alpha_{n} \leqslant C \\
\sum_{n=1}^{N} \alpha_{n} y_{n}=0 \quad, n=1, \ldots, N
\end{array}\right.
\end{aligned}
$$

It is still a quadratic programming problem, and can be easily solved. However, there is still an outstanding issue, i.e., the value of parameter $C$ and $\sigma$ in Eq. (8). 


\subsection{PSO}

To determine the best parameter of $C$ and $\sigma$, the traditional method uses either trial-and-error or gridsearching methods. They will cause heavy computation burden, and cannot guarantee to find the optimal or even near-optimal solutions. PSO is commonly used for training the KSVM.

PSO performs searching via a swarm of particles that updates from iteration to iteration. Each particle moves along the direction updated by both its previously best (pbest) position and the global best ( $g$ best) position in the swarm [28].

$$
\begin{aligned}
\operatorname{pbest}(i, t) & =\underset{k=1, \ldots, t}{\arg \min }\left[f\left(P_{i}(k)\right)\right], i \in\{1,2, \ldots, N\} \\
\operatorname{gbest}(t) & =\underset{\substack{i=1, \ldots, N \\
k=1, \ldots, t}}{\arg \min }\left[f\left(P_{i}(k)\right)\right]
\end{aligned}
$$

where $i$ denotes the particle index, $N$ the total number of particles, $t$ the current iteration number, $f$ the fitness function, and $P$ the position. The velocity $V$ and position $P$ of particles are updated by:

$$
\begin{aligned}
& V_{i}(t+1)=\omega V_{i}(t)+c_{1} r_{1}\left(\text { pbest }(i, t)-P_{i}(t)\right)+c_{2} r_{2}\left(\operatorname{gbest}(t)-P_{i}(t)\right) \\
& P_{i}(t+1)=P_{i}(t)+V_{i}(t+1)
\end{aligned}
$$

where $V$ denotes the velocity, $\omega$ the inertia weight used to balance the global exploration and local exploitation, $r_{1}$ and $r_{2}$ random numbers within range [0,1], $c_{1}$ and $c_{2}$ acceleration coefficients. "Velocity clamping" [29] was used to set an upper bound for the velocity parameter as a way to limit particles flying out of the search space.

\section{5. $Q P S O$}

The main disadvantage of PSO is that it is easily to trap into local optima, even if it converges in a very fast way [30]. Inspired by trajectory analysis and quantum mechanics, the quantum-behaved PSO (QPSO) is developed.

Assume the PSO is a quantum-like system, each single particle is spinless and has a quantum behavior formulated by the wave function without regard to the interference from other particles. Further, assume each individual particle moves in a delta potential well of the search space. van den Bergh and Engelbrecht [31] yield each particle converges to its local attractor $a_{i}$ defined as

$$
a_{i}=\frac{c_{1} \times \operatorname{pbest}(i)+c_{2} \times \text { gbest }}{c_{1}+c_{2}}
$$

In QPSO, both the solution space and the search space are totally different. The wave function, viz., the probability function, of position depicts in quantized search space the state of the particle, not offering any certain position information. Therefore, it is impossible to use wave function to evaluate the fitness. To solve it, state transformation between two spaces is required [32].

Note that the transformation from quantum state to classical state is termed "collapse", which is defined as the measurement of a particle position. Using Monte Carlo method, the positions of particles are:

$$
P_{i}(t+1)= \begin{cases}a_{i}(t)+\frac{L_{i}(t)}{2} \ln \left(\frac{1}{u}\right) & h>0.5 \\ a_{i}(t)-\frac{L_{i}(t)}{2} \ln \left(\frac{1}{u}\right) & \text { otherwise }\end{cases}
$$


Table 1

Classifier comparison $(\mathrm{WE}=$ Wavelet-energy)

\begin{tabular}{|c|c|c|c|c|c|}
\hline & Success cases & Sensitivity & Specificity & Precision & Accuracy \\
\hline \multicolumn{6}{|l|}{ Existing methods } \\
\hline $\mathrm{DWT}+\mathrm{PCA}+\mathrm{BP}-\mathrm{NN}$ [15] & 388 & $88.0 \%$ & $56.00 \%$ & $97.14 \%$ & $86.22 \%$ \\
\hline DWT + PCA + RBF-NN [15] & 411 & $92.47 \%$ & $72.00 \%$ & $98.25 \%$ & $91.33 \%$ \\
\hline $\mathrm{DWT}+\mathrm{PCA}+\mathrm{PSO}-\mathrm{KSVM}[15]$ & 440 & $98.12 \%$ & $92.00 \%$ & $99.52 \%$ & $97.78 \%$ \\
\hline $\mathrm{WE}+\mathrm{BP}-\mathrm{NN}[35]$ & 390 & $88.47 \%$ & $56.00 \%$ & $97.16 \%$ & $86.67 \%$ \\
\hline WE + KSVM [36] & 413 & $93.18 \%$ & $68.00 \%$ & $98.02 \%$ & $91.78 \%$ \\
\hline $\mathrm{DWT}+\mathrm{PCA}+\mathrm{GA}-\mathrm{KSVM}[20]$ & 439 & $97.88 \%$ & $92.00 \%$ & $99.52 \%$ & $97.56 \%$ \\
\hline WE + PSO-KSVM [21] & 437 & $97.65 \%$ & $88.00 \%$ & $99.28 \%$ & $97.11 \%$ \\
\hline $\mathrm{WE}+\mathrm{BBO}-\mathrm{KSVM}[21]$ & 440 & $98.12 \%$ & $92.00 \%$ & $99.52 \%$ & $97.78 \%$ \\
\hline \multicolumn{6}{|l|}{ Proposed methods } \\
\hline $\mathrm{WE}+\mathrm{QPSO}-\mathrm{KSVM}$ & 442 & $98.59 \%$ & $92.00 \%$ & $99.52 \%$ & $98.22 \%$ \\
\hline
\end{tabular}

where $u$ and $h$ in the range $[0,1]$ are random numbers. $L$ depends on energy intension of the potential well, specifying the search range of a particle and is called creativity of the particle. Its form is

$$
L_{i}=2 \beta \mid \text { mbest }-P_{i} \mid
$$

where $\beta$ is called the creativity coefficient (or contraction-expansion coefficient), and $m$ best denotes the mean best of the population (or Mainstream Thought), with the definition of the average of the $p$ best positions in the swarm:

$$
\operatorname{mbest}(t)=\frac{1}{N} \sum_{i=1}^{N} \operatorname{pbest}_{i}(t)
$$

Equation (14) is the main iterative procedures in the QPSO algorithm [33].

\section{Experiments and discussions}

\subsection{Feature extraction}

The 3-level 2D-DWT of Haar wavelet decomposed the input image into 10 subbands as shown in Fig. 1. Symmetric padding method [34] was utilized to calculate the boundary value, with the aim of avoiding border distortion. After decomposition, 10 features were obtained from calculating the energy of those 10 subbands (LL3, HL3, LH3, HH3, HH2, HL2, LH2, HH1, HL1, and LH1). The waveletenergy can reduce the dimension of DWT coefficients. For a $256 \times 256$ image, the 3-level DWT did not reduce the dimension, but the 3-level wavelet-energy can reduce the feature dimension from 65,536 to only 10 .

\subsection{Algorithm comparison}

The wavelet-energy features were fed into different classifiers. We compared the proposed "WE + QPSO-KSVM" method with "DWT + PCA + BP-NN [15]", "DWT + PCA + RBF-NN [15]", "DWT + PCA + PSO-KSVM [15]", "WE + BPNN [35]", "WE + KSVM [36]", and "DWT + PCA + GAKSVM [20]". The results were shown in Table 1. The kernel function was set to RBF. 


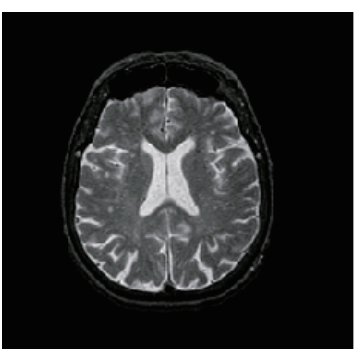

(a)

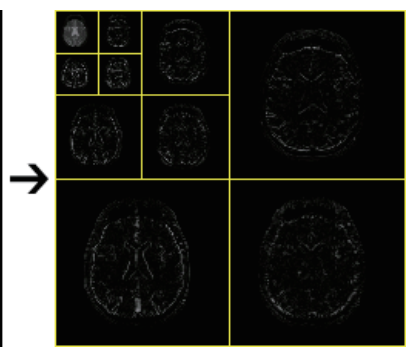

(b)

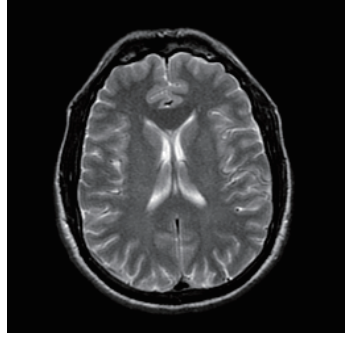

(a)

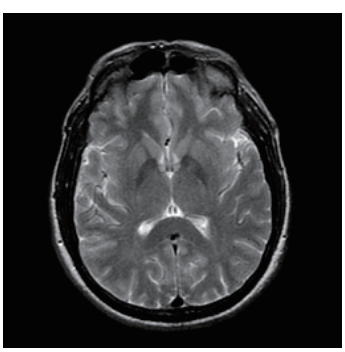

(b)

Fig. 1. Illustration of DWT: (a) a normal brain image; (b) its 3-level 2D DWT decomposition result.

Fig. 2. Two images of Lyme Encephalopathy: (a) Incorrectly classified; (b) Correctly classified.

Results in Table 1 showed that the proposed "WE + QPSO-KSVM" correctly matched 442 cases with accuracy of sensitivity of $98.59 \%$, specificity of $92.00 \%$, precision of $99.52 \%$, and accuracy of $98.22 \%$. The results are superior to state-of-the-art algorithms.

Why the proposed QPSO-KSVM performs the best? Because it is combined by two successful components: the KSVM and the QPSO. KSVM is widely used for pattern-recognition problems, and it is reported to achieve excellent results. The importance of introducing QPSO is to determine the optimal values of parameters $C$ and $\sigma$ in KSVM, otherwise it is difficult to get the optimal weights for KSVM. QPSO is a relatively novel global optimization method that was inspired by trajectory analysis and quantum mechanics. Integrating QPSO to KSVM enhances the classification capability of KSVM. The comparison in Table 1 showed the effectiveness of QPSO.

\subsection{Fail cases}

Two images of Lyme Encephalopathy are shown in Fig. 2. The left image was incorrectly classified as normal, and the right image was correctly predicted as abnormal. The reason is the left image did not fully capture the focus and deformed region. This gives us a hint that the proposed method may be improved by using multi-slices.

\section{Conclusions and future research}

The contributions of this paper lie in following points: (i) We proposed a new approach for automatic abnormal brain detection using Wavelet-Energy, KSVM, and QPSO. (ii) The experiments demonstrated the proposed method performed better than existing methods. (iii) Wavelet-Energy was validated as effective feature for abnormal brain detection.

The excellent classification performance suggested that the proposed system may be used in practical CAD to help physicians to make decisions whether the brain is normal or abnormal. Further, this CAD system may be used for MR brain image classification with different pathological types, condition, and statuses.

In the future, we may focus on following regards: (1) we may replace wavelet-energy with more efficient feature descriptors, such as scale-invariant features. (ii) Some advanced pattern recognition techniques may be used, such as deep learning and RBFNN [37]. (iii) Multiple slices can be used to improve the classification performance. 


\section{Acknowledgments}

This paper was supported by NSFC (610011024, 61273243 and 51407095), Program of Natural Science Research of Jiangsu Higher Education Institutions (13KJB460011 and 14KJB520021), Jiangsu Key Laboratory of 3D Printing Equipment and Manufacturing (BM2013006), Key Supporting Science and Technology Program (Industry) of Jiangsu Province (BE2012201, BE2014009-3 and BE20130122), Special Funds for Scientific and Technological Achievement Transformation Project in Jiangsu Province (BA2013058), Nanjing Normal University Research Foundation for Talented Scholars (2013119XGQ0061 and 2014119XGQ0080). Open Project Program of the State Key Lab of CAD\&CG, Zhejiang University (A1616), Open Project Program of Key laboratory of symbolic computation and knowledge engineering of ministry of education, Jilin University (93K172016K17), Open Fund of Guangxi Key Laboratory of Manufacturing System \& Advanced Manufacturing Technology (15-14030-008K).

\section{Conflict of interest}

We have no conflicts of interest to disclose with regard to the subject matter of this paper.

\section{References}

[1] Y.D. Zhang, Z.C. Dong, G.L. Ji and S.H. Wang, An improved reconstruction method for CS-MRI based on exponential wavelet transform and iterative shrinkage/thresholding algorithm. Journal of Electromagnetic Waves and Applications 28, 2327 (2014).

[2] S. Goh, Z. Dong, Y. Zhang, S. DiMauro and B.S. Peterson, Mitochondrial dysfunction as a neurobiological subtype of autism spectrum disorder: Evidence from brain imaging. JAMA Psychiatry 71, 665 (2014).

[3] Y. Zhang, S. Wang, G. Ji and Z. Dong, An improved quality guided phase unwrapping method and its applications to MRI. Progress in Electromagnetics Research 145, 273 (2014).

[4] F. Thorsen, B. Fite, L.M. Mahakian, J.W. Seo, S.P. Qin, V. Harrison, S. Johnson, E. Ingham, C. Caskey, T. Sundstrom, T.J. Meade, P.N. Harter, K.O. Skaftnesmo and K.W. Ferrara, Multimodal imaging enables early detection and characterization of changes in tumor permeability of brain metastases. Journal of Controlled Release 172, 812 (2013).

[5] P. Maji, M.K. Kundu and B. Chanda, Second order fuzzy measure and weighted co-occurrence matrix for segmentation of brain MR images. Fundam Inform 88, 161 (2008).

[6] E.S.A. El-Dahshan, T. Hosny and A.B.M. Salem, Hybrid intelligent techniques for MRI brain images classification. Digital Signal Processing 20, 433 (2010).

[7] Y. Zhang, L. Wu and S. Wang, Magnetic resonance brain image classification by an improved artificial bee colony algorithm. Progress in Electromagnetics Research 116, 65 (2011).

[8] Y. Zhang, Z. Dong, L. Wu and S. Wang, A hybrid method for MRI brain image classification. Expert Systems with Applications 38, 10049 (2011).

[9] R. Ramasamy, and P. Anandhakumar, Brain tissue classification of MR images using fast Fourier transform based expectation-maximization Gaussian mixture model, Advances in Computing and Information Technology, Springer (2011), pp. 387-398.

[10] Y. Zhang and L. Wu, An MR brain images classifier via principal component analysis and kernel support vector machine. Progress in Electromagnetics Research 130, 369 (2012).

[11] M. Saritha, K. Paul Joseph and A.T. Mathew, Classification of MRI brain images using combined wavelet entropy based spider web plots and probabilistic neural network. Pattern Recognition Letters 34, 2151 (2013).

[12] Y. Zhang, Z. Dong, G. Ji and S. Wang, Effect of spider-web-plot in MR brain image classification. Pattern Recognition Letters 62, 14 (2015).

[13] S. Das, M. Chowdhury and M.K. Kundu, Brain MR image classification using multiscale geometric analysis of ripplet. Progress in Electromagnetics Research-Pier 137, 1 (2013).

[14] H. Kalbkhani, M.G. Shayesteh and B. Zali-Vargahan, Robust algorithm for brain magnetic resonance image (MRI) classification based on GARCH variances series. Biomedical Signal Processing and Control 8, 909 (2013). 
[15] Y. Zhang, S. Wang, G. Ji and Z. Dong, An MR brain images classifier system via particle swarm optimization and kernel support vector machine. The Scientific World Journal 2013, 9 (2013).

[16] A. Padma and R. Sukanesh, Segmentation and classification of brain CT images using combined wavelet statistical texture features. Arab J Sci Eng 39, 767 (2014).

[17] E.S.A. El-Dahshan, H.M. Mohsen, K. Revett and A.B.M. Salem, Computer-aided diagnosis of human brain tumor through MRI: A survey and a new algorithm. Expert Systems with Applications 41, 5526 (2014).

[18] X. Zhou, S. Wang, W. Xu, G. Ji, P. Phillips, P. Sun and Y. Zhang, Detection of pathological brain in MRI scanning based on wavelet-entropy and naive Bayes classifier, Bioinformatics and Biomedical Engineering, edited by F. Ortuño and I. Rojas, Springer International Publishing (2015), pp. 201-209.

[19] Y. Zhang, Z. Dong, S. Wang, G. Ji and J. Yang, Preclinical diagnosis of magnetic resonance (MR) brain images via discrete wavelet packet transform with tsallis entropy and generalized eigenvalue proximal support vector machine (GEPSVM). Entropy 17, 1795 (2015).

[20] S. Wang, G. Ji, P. Phillips and Z. Dong, Application of genetic algorithm and kernel support vector machine to pathological brain detection in MRI Scanning. The 2nd National Conference on Information Technology and Computer Science (2015), pp. 450-456.

[21] G. Yang, Y. Zhang, J. Yang, G. Ji, Z. Dong, S. Wang, C. Feng and Q. Wang, Automated classification of brain images using wavelet-energy and biogeography-based optimization. Multimedia Tools and Applications 1, (2015). doi: $10.1007 / \mathrm{s} 11042-015-2649-7$.

[22] S. Wang, Y. Zhang, Z. Dong, S. Du, G. Ji, J. Yan, J. Yang, Q. Wang, C. Feng and P. Phillips, Feed-forward neural network optimized by hybridization of PSO and ABC for abnormal brain detection. International Journal of Imaging Systems and Technology 25, 153 (2015).

[23] Y. Zhang, Z. Dong, P. Phillips, S. Wang, G. Ji, J. Yang and T.-F. Yuan, Detection of subjects and brain regions related to Alzheimer's disease using 3D MRI scans based on eigenbrain and machine learning. Frontiers in Computational Neuroscience 66, 1 (2015).

[24] S.H. Lee, C.K. Lee, J.B. Park and Y.H. Choi, Diagnostic method for insulated power cables based on wavelet energy. IEICE Electronics Express 10, (2013). pp. 335-335.

[25] T.Y. Yaroshenko, D.V. Krysko, V. Dobriyan, M.V. Zhigalov, H. Vos, P. Vandenabeele and V.A. Krysko, Wavelet modeling and prediction of the stability of states: the Roman Empire and the European Union. Communications in Nonlinear Science and Numerical Simulation 26, 265 (2015).

[26] Y.L. Wu, C.T. Yeh, W.C. Hung and C.Y. Tang, Gaze direction estimation using support vector machine with active appearance model. Multimedia Tools and Applications 70, 2037 (2014).

[27] P.J.G. Nieto, E. Garcia-Gonzalo, F.S. Lasheras and F.J.D. Juez, Hybrid PSO-SVM-based method for forecasting of the remaining useful life for aircraft engines and evaluation of its reliability. Reliability Engineering \& System, Safety 138, 219 (2015).

[28] Y. Zhang, S. Balochian, P. Agarwal, V. Bhatnagar and O.J. Housheya, Artificial intelligence and its applications. Mathematical Problems in Engineering 2014, 10 (2014).

[29] F. Shahzad, S. Masood and N.K. Khan, Probabilistic opposition-based particle swarm optimization with velocity clamping. Knowledge and, Information Systems 39, 703 (2014).

[30] L. Lin, F. Guo, X.L. Xie and B. Luo, Novel adaptive hybrid rule network based on TS fuzzy rules using an improved quantum-behaved particle swarm optimization. Neurocomputing 149, 1003 (2015).

[31] F. van den Bergh and A.P. Engelbrecht, A study of particle swarm optimization particle trajectories. Information Sciences 176, 937 (2006).

[32] E. Davoodi, M.T. Hagh and S.G. Zadeh, A hybrid improved quantum-behaved particle swarm optimization-simplex method (IQPSOS) to solve power system load flow problems. Applied Soft Computing 21, 171 (2014).

[33] X. Fu, W.S. Liu, B. Zhang and H. Deng, Quantum behaved particle swarm optimization with neighborhood search for numerical optimization. Mathematical Problems in Engineering (2013), vol. 2013, doi: 10.1155/2013/469723.

[34] A. Messina, Refinements of damage detection methods based on wavelet analysis of dynamical shapes. International Journal of Solids and Structures 45, 4068 (2008).

[35] R. Choudhary, S. Mahesh, J. Paliwal and D.S. Jayas, Identification of wheat classes using wavelet features from near infrared hyperspectral images of bulk samples. Biosystems Engineering 102, 115 (2009).

[36] M.R.K. Mookiah, U.R. Acharya, C.M. Lim, A. Petznick and J.S. Suri, Data mining technique for automated diagnosis of glaucoma using higher order spectra and wavelet energy features. Knowledge-Based Systems 33, 73 (2012).

[37] D.L. Guo, Y.D. Zhang, Q. Xiang and Z.H. Li, Improved radio frequency identification indoor localization method via radial basis function neural network. Mathematical Problems in Engineering (2014), vol. 2014, doi: 10.1155/2014/420482. 